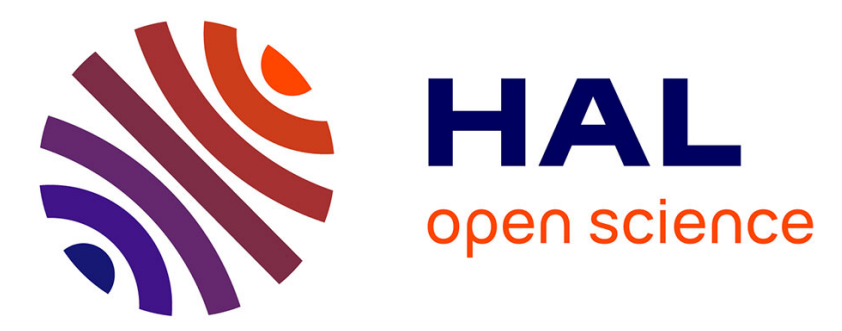

\title{
Modulation d'un effet thermomagnétique à basse température et détection par le deuxième son dans l'hélium
}

\author{
F. Vidal, Y. Simon, M. Le Ray, P. Thorel
}

\section{- To cite this version:}

F. Vidal, Y. Simon, M. Le Ray, P. Thorel. Modulation d'un effet thermomagnétique à basse température et détection par le deuxième son dans l'hélium. Revue de Physique Appliquée, 1969, 4 (1), pp.50-56. 10.1051/rphysap:019690040105000 . jpa-00242890

\section{HAL Id: jpa-00242890 https://hal.science/jpa-00242890}

Submitted on 1 Jan 1969

HAL is a multi-disciplinary open access archive for the deposit and dissemination of scientific research documents, whether they are published or not. The documents may come from teaching and research institutions in France or abroad, or from public or private research centers.
L'archive ouverte pluridisciplinaire HAL, est destinée au dépôt et à la diffusion de documents scientifiques de niveau recherche, publiés ou non, émanant des établissements d'enseignement et de recherche français ou étrangers, des laboratoires publics ou privés. 


\title{
MODULATION D'UN EFFET THERMOMAGNÉTIQUE A BASSE TEMPÉRATURE ET DÉTEGTION PAR LE DEUXIÈME SON DANS L'HÉLIUM
}

\author{
Par F. VIDAL, Y. SIMON, M. LE RAY ( $\left.{ }^{1}\right)$ et P. THOREL $\left({ }^{2}\right)$, \\ Groupe de Physique des Solides de l'École Normale Supérieure $\left({ }^{3}\right)$, Paris, $5^{\mathrm{e}}$. \\ (Reçu le 12 décembre 19j8.)
}

\begin{abstract}
Résumé. - En calorimétrie, et dans l'étude de divers effets thermiques, à très basse température, de nombreux auteurs utilisent de préférence à une méthode en continu une technique de modulation du flux de chaleur avec détection synchrone des écarts thermiques ; l'échantillon, au lieu d'être isolé, est en faible contact thermique avec le bain d'hélium, et la fréquence de modulation est de l'ordre de 5 à $10 \mathrm{~Hz}$. Nous proposons une autre méthode de modulation qui permet de travailler à des fréquences plus élevées de la gamme acoustique, pourvu que l'amplitude du flux de chaleur soit suffisante, par exemple, dans notre cas, supérieure ou égale à $10^{-5} \mathrm{~W} / \mathrm{cm}^{2}$. L'échantillon est mis en contact direct avec un bain d'hélium superfluide; le flux de chaleur est dérivé en partie vers l'hélium pour engendrer une onde de deuxième son. Cette onde est amplifiée par un tuyau résonnant $(Q=125$ à $300 \mathrm{~Hz})$ fermé d'une part par l'échantillon émetteur et d'autre part par un bolomètre récepteur. Nous avons appliqué cette méthode à l'étude de l'effet Etttingshausen dans un supraconducteur de deuxième espèce.
\end{abstract}

Abstract. - In calorimetry and in the study of different types of thermal effects, at very low temperatures, several authors have preferred to use a heat current modulation technique along with synchronous detection of the thermal amplitudes rather than a steady heat current technique. Instead of being isolated, the sample and the bath are coupled through a weak heat-leak and the modulating frequency ranges from 5 to $10 \mathrm{C} / \mathrm{s}$. Another method of modulation is proposed which enables us to work at higher frequencies in the audio-range, provided the heat current amplitude is sufficient, for instance, in our case, greater than $10^{-5} \mathrm{~W} / \mathrm{cm}^{2}$. The sample is in direct contact with a bath of superfluid helium; the heat current is partly diverted into the helium and creates a second sound wave. This wave is amplified by a resonant pipe $(Q=125$ at $300 \mathrm{C} / \mathrm{s}$ ) shut at one end by the sample and at the other end by a receiver. This method has been applied to the study of the Ettingshausen effect in a type II superconductor.

I. Introduction. - L'usage de bolomètres collés sur les échantillons est assez général pour l'étude des effets thermiques dans les solides à très basse température, qu'il s'agisse d'effets étudiés pour eux-mêmes (effets thermoélectriques et thermomagnétiques [1], effet magnétothermique [2], calorimétrie [3]) ou d'effets secondaires mesurant une absorption d'énergie (résonance cyclotron [4]). D'autre part, les auteurs $[2,3,4]$ préfèrent en général aux méthodes d'excitation continue des méthodes d'excitation alternative en basse fréquence qui permettent une détection synchrone.

Nous présentons ici une méthode originale dont le principe est de créer, en modulant la source excitatrice, une onde de deuxième son dans l'hélium II $(T<2,17 \mathrm{~K})$. Cette onde est amplifiée dans un tuyau

(1) Adresse permanente : Laboratoire de Mécanique des Fluides, Faculté des Sciences, Orsay.

(2) Laboratoire central de la C.S.F., 91-Corbeville.

(3) Laboratoire associé au Centre National de la Recherche Scientifique. à ondes stationnaires (cf. § III) accordé à la fréquence de travail et elle est détectée par un bolomètre placé à distance de l'échantillon. Le bolomètre et l'échantillon constituent les deux extrémités fermées de ce tuyau (cf. $§ I V)$.

Nous discuterons la possibilité d'appliquer cette méthode à l'étude d'un effet thermomagnétique : l'effet Ettingshausen dans un supraconducteur de type II, qui est particulièrement marqué dans l'état dit tourbillonnaire [1]. Cet exemple nous permettra de préciser dans quelles conditions expérimentales elle peut avoir un intérêt.

Landau [5] a développé la théorie phénoménologique de l'hélium superfluide, basée sur le modèle à deux fluides : en dessous du point $\lambda$, à une température $T$, existent en équilibre, dans une certaine proportion, fonction de $T$, un fluide " normal » doué de viscosité et d'entropie et un « superfluide » sans viscosité et sans entropie, qui a les propriétés d'un fluide parfait, soit pour des mouvements assez lents, soit pour 
des fréquences assez élevées (cas du $2^{\mathrm{e}}$ son). Aux divers mouvements possibles de ces deux fluides relativement l'un à l'autre et relativement aux parois, correspondent divers modes de propagation d'ondes.

En laissant de côté les cas où les dimensions transversales offertes au fluide normal sont si faibles qu'elles empêchent pratiquement celui-ci d'osciller ( 3 e et $4^{\mathrm{e}}$ sons resp. dans les films et les pores d'une poudre fine), on distingue dans le liquide « massif » deux modes simples de vibration :

a) Le premier son, lorsque les deux fluides se déplacent en phase et à la même vitesse (son ordinaire);

b) Le deuxième son, lorsque les deux fluides se déplacent en opposition de phase et sans transport global de masse $\left(\rho_{\mathrm{n}} \mathbf{V}_{\mathrm{n}}+\rho_{\mathrm{s}} \mathbf{V}_{\mathrm{s}}=0\right)$; dans ce cas, il n'y a aucune oscillation de pression ou de densité, mais une oscillation d'entropie spécifique, donc de température.

Pour exciter le second son, on provoquera sur une surface solide immobile, en contact direct avec l'hélium, une variation de température. Le plus souvent, l'émetteur est une résistance (ruban ou film résistant de faible capacité thermique) parcourue par un courant alternatif de fréquence $\omega$, d'où un effet Joule et une onde $2^{\mathrm{e}}$ son à la fréquence $2 \omega$ [6]. D'autres émetteurs ont été proposés; citons Peshkov [7] qui, à l'aide d'un superfiltre, transforme un $1^{\mathrm{er}}$ son en $4^{\mathrm{e}}$ son (dans le superfiltre), puis en second son, ainsi que Kurti et Mac Intosh [8] qui utilisent un sel paramagnétique plongé dans un champ alternatif.

Pour détecter le $2^{\mathrm{e}}$ son, on emploie une résistance de très faible capacité thermique (film ou fil très fin) sensible à la température locale. Si on polarise la résistance par un courant continu, on mesurera une tension alternative à ses bornes.

$$
\left[\begin{array}{c}
\frac{\nabla_{x} \mu}{e} \\
\frac{\nabla_{y} \mu}{e} \\
q_{x} \\
q_{y}
\end{array}\right]=\left[\begin{array}{cc}
L_{11} & L_{\mathbf{1 2}} \\
-L_{12} & L_{11} \\
-T L_{13} & -T L_{14} \\
T L_{14} & -T L_{13}
\end{array}\right.
$$

Dans la matrice $L_{i j}$, il y a six coefficients cinétiques indépendants fonction du champ $B$.

L'échantillon étudié est une lame parallélépipédique d'épaisseur $e$ dans la direction $y$ (fig. 1). Un courant électrique est injecté dans la direction $x$ avec une densité $j_{x}$ que nous supposerons uniforme. Ici donc, $j_{y}=0$. Nous nous intéresserons au flux de chaleur dans la direction $y$ (direction d'émission du second son) :

$$
q_{y}=T L_{14} j_{x}+L_{34} \nabla_{x} T-L_{33} \nabla_{y} T .
$$

Nous négligerons le terme d'effet Hall thermique $L_{34} \nabla_{x} T$ (Righi-Leduc), d'autant plus que $\nabla_{x} T$ est
II. Effet thermomagnétique et analogie électrique. - Les effets thermomagnétiques apparaissent dans un solide parcouru par des courants électriques $\mathbf{j}$ et (ou) des flux de chaleur q (dans le plan $x y$ ), en présence d'un champ magnétique $B$ (appliqué dans la direction $z$ ) (fig. 1). Le gradient du potentiel électrochi-

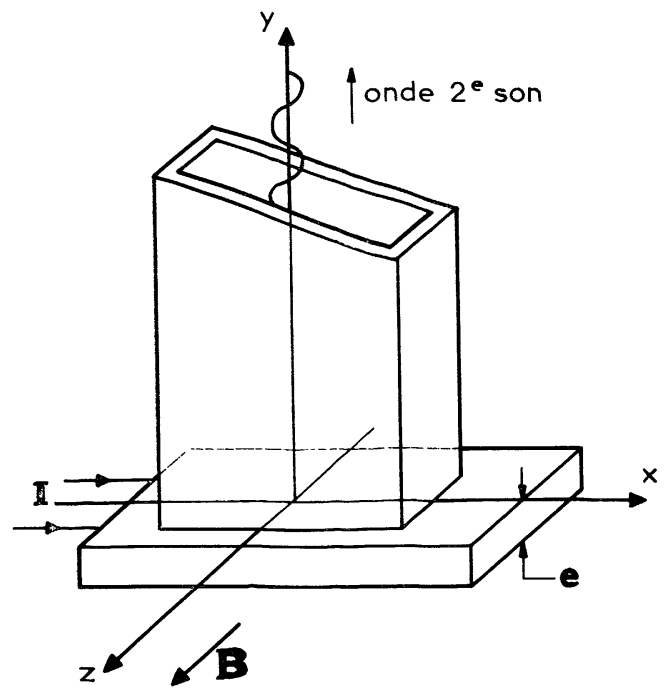

FIG. 1. - Schéma de l'échantillon et du tuyau résonnant.

mique $\mu\left(\nabla_{x} \mu, \nabla_{y} \mu\right)$, le gradient de température $\left(\nabla_{x} T, \nabla_{y} T\right)$, la densité de courant $\mathbf{j}\left(j_{x}, j_{y}\right)$ et le flux de chaleur $\mathbf{q}\left(q_{x}, q_{y}\right)$ sont reliés par des relations linéaires qui généralisent la loi d'Ohm et la loi de conduction de la chaleur, et qu'on peut écrire sous la forme pratique [9] :

$\left.\begin{array}{rr}L_{13} & L_{14} \\ -L_{14} & L_{13} \\ L_{33} & L_{34} \\ -L_{34} & L_{33}\end{array}\right] \cdot\left[\begin{array}{c}j_{x} \\ j_{y} \\ -\nabla_{x} T \\ -\nabla_{y} T\end{array}\right]$.

faible devant $\nabla_{y} T$ pour un échantillon très allongé dans la direction $x$ et plongé dans un milieu homogène (ici ce sera l'hélium II) :

$$
q_{y}=T L_{14} j_{x}-L_{33} \nabla_{y} T .
$$

Si $q_{y}=0$, l'échantillon étant isolé thermiquement sur les faces $x z$ (par exemple le vide; voir Otter et Solomon [1]), on écrit $T L_{14} j_{x}=L_{33} \nabla_{y} T$ et on dit que le flux d'entropie $L_{\mathbf{1 4}} j_{x}$, provoqué à angle droit par le courant $j_{x}$, est compensé par un flux de conduction opposé $L_{33} \nabla_{y} T, L_{33}$ correspondant au coefficient thermique habituel $K$. 
$\mathrm{Si}$, au contraire, on réalise des conditions isothermes, par des contacts thermiques parfaits sur les faces $x z$, $\nabla_{y} T=0$, et alors $q_{y}=T L_{\mathbf{1 4}} j_{x}$ et tout le flux $T L_{14} j_{c}$ passe dans le milieu environnant.

Nous verrons que nos conditions expérimentales correspondent à une situation intermédiaire entre ces deux cas extrêmes. Nous l'appellerons l'effet Ettingshausen, bien que celui-ci soit défini strictement par la condition $q_{y}=0 . L_{14}$ est un coefficient impair en $B$, tandis que $L_{33}$ peut avoir des variations en $B^{2}$.

Dans le cas d'un régime variable avec le temps, $q_{y}$ et $T$ sont fonction de $y$ et $t$ (problème à 1 dimension). On écrira toujours l'équation (2), puis la conservation de la chaleur :

$$
-\frac{\partial q_{y}}{\partial y}+G=\gamma \frac{\partial T}{\partial t}
$$

où $G$ est le taux de production de chaleur par unité de volume, en particulier l'effet Joule.

En régime harmonique, à une fréquence $\omega$, on impose le courant $j_{x}=j \mathrm{e}^{i \omega t}+j_{0} . j$ est constant, si $\omega$ est assez faible pour que l'effet de peau électrique soit négligeable sur l'épaisseur $e . j_{0}$ tient compte d'un courant continu éventuellement superposé. Dans ces conditions, posons $T L_{\mathbf{1 4}} j_{x}=T L_{\mathbf{1 4}} j_{\mathbf{0}}+q_{1} \mathrm{e}^{i \omega t}$. S'il y a un effet Joule normal en $j_{x}^{2}$, il apparaît en $G$ un terme continu $g_{0}$, un terme $g_{1} \mathrm{e}^{i \omega t}$ à la fréquence fondamentale que nous appelons effet Joule croisé, et un terme $g_{2} \mathrm{e}^{2 i \omega t}$. Les composantes fondamentales de $T$ et $q_{y}\left(\Delta T \mathrm{e}^{i \omega t}, q \mathrm{e}^{i \omega t}\right)$ sont données alors par les deux équations linéaires :

$$
\begin{gathered}
q=q_{1}-L_{33} \frac{\mathrm{d} \Delta T}{\mathrm{~d} y} \\
-\frac{\mathrm{d} q}{\mathrm{~d} y}+g_{1}=i \gamma \omega \Delta T
\end{gathered}
$$

$q, q_{1}$ sont en $\mathrm{W} / \mathrm{cm}^{2}, g_{1}$ en $\mathrm{W} / \mathrm{cm}^{3}, \Delta T$ en ${ }^{\circ} \mathrm{K}, L_{33}$ en $\mathrm{W} / \mathrm{cm}{ }^{\circ} \mathrm{K}$ et $\gamma$ en $\mathrm{J} / \mathrm{cm}^{3}$ oK.

A $\left(2^{\prime}\right)$ et $\left(3^{\prime}\right)$, il faut ajouter les conditions aux limites sur les faces :

$$
\begin{gathered}
q(-e)=-\Delta T(-e) / Z \\
q(0)=\Delta T(0) / Z
\end{gathered}
$$

où $Z\left(\right.$ en $\left.\mathrm{cm}^{2} \mathrm{oK} / \mathrm{W}\right)$ est la résistance ou l'impédance de fuite vers le milieu environnant. Nous verrons que si l'échantillon est plongé dans l'hélium II, $Z$ égale pratiquement la résistance de contact avec l'hélium, $R_{\mathrm{K}}$, dite résistance Kapitza $\left(R_{\mathrm{K}} \sim 2\right.$ à 5 pour les métaux; cf. § III).

La figure 2 a représente l'échantillon suivant l'analogie électrique habituelle des équations de la chaleur. La lame dans la direction $y$ est analogue à une ligne dont la résistance et la capacité par unité de longueur sont respectivement $r=1 / L_{33}$ et $\gamma$, fermée aux deux extrémités sur des impédances $Z$. Un courant $-q_{1} \mathrm{e}^{i \omega t}$ est injecté à l'entrée $(y=0)$ et repris à la sortie $(y=e)$ (source de courant - $q_{1}$ branchée aux bornes). Éventuellement (cas de l'effet Joule), on injecte tout le long de la ligne une intensité $g_{1} \mathrm{e}^{i \omega t}$ par
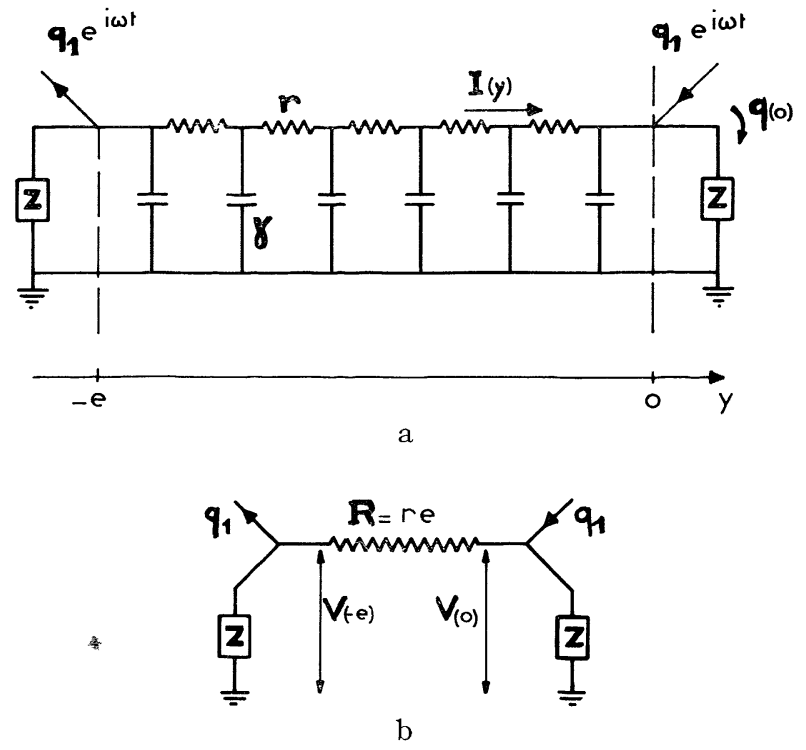

FIG. 2. - Représentation analogique d'un échantillon de résistance thermique $r$ et de capacité thermique $\gamma$ par unité de longueur :

a) Effet Ettingshausen alternatif.

b) En continu.

unité de longueur. Il en résulte une répartition de courant $I(y) \mathrm{e}^{i \omega t}$ et de tension $V(y) \mathrm{e}^{i \omega t}$ le long de la ligne qui sont identiques aux solutions $I(y)=q-q_{1}$ et $V(y)=\Delta T$ des équations $\left(2^{\prime}\right),\left(3^{\prime}\right)$ et $(4)$.

$V(y)=\Delta T$ et $I(y)=q-q_{1}$ obéissent aux équations d'onde :

$$
\begin{gathered}
\frac{\mathrm{d}^{2} I}{\mathrm{~d} y^{2}}-k^{2} I=0 \\
\frac{\mathrm{d}^{2} V}{\mathrm{~d} y^{2}}-k^{2} V=-r g_{1}
\end{gathered}
$$

avec les conditions aux limites : $I=\mp V / Z-q_{1}$. On a posé $k^{2}=i r \gamma \omega=2 i / \delta^{2}$ où $\delta$ est la profondeur de l'effet de peau thermique, dont il faut tenir compte dès que $\delta(\omega)$ devient de l'ordre de $e$.

On trouve que le flux de chaleur sortant par la face $y=0$ est une fonction linéaire des sources, $q_{1}$ et $g_{1} e\left(\mathrm{en} \mathrm{W} / \mathrm{cm}^{2}\right)$, flux thermomagnétique et flux total d'effet Joule respectivement :

$$
q(0)=I(0)=\alpha q_{1}+\beta\left(g_{1} e\right)
$$

$q_{1}$ et $g_{1} e$ ont la phase (zéro) du courant électrique $j_{x}, \alpha$ et $\beta$ sont en général deux nombres complexes fonction des nombres $e / \delta$ et $Z / R$, où $R=e r$ est la résistance thermique totale de l'échantillon (par $\left.\mathrm{cm}^{2}\right)$.

Quand $\omega \rightarrow 0 \quad(\delta \rightarrow \infty)$, cas limite du courant continu (fig. 2 b) :

$$
\alpha \rightarrow \frac{1}{1+2 Z / R} ; \quad \beta \rightarrow 1 / 2 .
$$


L'observation habituelle de l'effet Ettingshausen, en continu, correspond au cas de l'échantillon parfaitement isolé (vide) : $Z \rightarrow \infty$, et on observe la différence de température :

$$
\Delta T=V(0)-V(-e)=R q_{1} .
$$

On aurait un effet isotherme seulement si $Z \rightarrow 0$, c'est-à-dire pratiquement quand $Z \ll R$, condition qui n'est pas forcément réalisée au contact de l'hélium II (§ III).

III. Résonateur de deuxième son. - Nous précisons en particulier dans ce paragraphe la relation entre le facteur $Q$ et le facteur d'amplification, notions souvent mal définies dans la littérature.

L'échantillon émetteur est baigné dans l'hélium et vient fermer une extrémité d'un tuyau (problème à une dimension). En principe, si le tuyau n'est pas limité, le flux de chaleur $q(0)$ (voir $(6)$ ), qui attaque l'hélium à travers la résistance de contact $R_{\mathrm{K}}$ (localisée à l'interface solide-hélium), va engendrer une onde plane progressive de température [10] se propageant dans la direction $y$ :

$$
\begin{aligned}
\Delta T=V(y, t)=q(0) z_{\infty} \mathrm{e}^{i(\omega t-K y)} & \\
& =q(0) z_{\infty} \mathrm{e}^{-\alpha y} \mathrm{e}^{i \omega(t-z / u)}
\end{aligned}
$$

où $z_{\infty}$ est l'impédance caractéristique de l'hélium, $u$ la vitesse du deuxième son, $\alpha$ un coefficient d'atténuation $(K=\omega / u-i \alpha)$. La ligne de la figure 2 se trouve donc fermée sur $Z=R_{\mathrm{K}}+z_{\infty}$.

Si $\rho_{n}$ et $\rho_{\mathrm{s}}$ sont les masses volumiques des deux fluides normal et superfluide $\left(\rho_{\mathrm{n}}+\rho_{\mathrm{s}}=\rho\right), S$ et $C_{\mathrm{v}}$ l'entropie et la chaleur spécifiques, on montre que [10]:

$$
u^{2}=\frac{\rho_{\mathrm{s}}}{\rho_{\mathrm{n}}} \frac{S^{2} T}{C_{\mathrm{v}}} .
$$

Si $\alpha$ est négligeable, une colonne d'hélium est analogue à une ligne sans pertes ayant une self $\lambda$ et une capacité $\gamma$ par unité de longueur [11] :

$$
\lambda=\frac{1}{\rho C_{\mathrm{v}} u^{2}} ; \quad \gamma=\rho C_{\mathrm{v}} .
$$

On sait que la vitesse de propagation le long d'une telle ligne est bien $u=\sqrt{1 / \lambda \gamma}$ et que son impédance caractéristique est une résistance pure :

$$
z_{\infty}=\sqrt{\lambda / \gamma}=1 / \rho C_{\mathrm{v}} u
$$

Si $\propto$ n'est pas négligeable, il faut ajouter des résistances série ou parallèle dans la ligne équivalente.

A $1,63 \circ \mathrm{K}, u=20,3 \mathrm{~m} / \mathrm{s}, z_{\infty} \simeq 2 \times 10^{-3}\left({ }^{\circ} \mathrm{K} \mathrm{cm} \mathrm{cm}^{2} / \mathrm{W}\right)$. On voit que $Z=R_{\mathrm{K}}+z_{\infty}$ est pratiquement égal à $R_{\mathrm{K}}(\sim 1)$. A un flux de $1 \mathrm{~mW} / \mathrm{cm}^{2}$ correspondent des amplitudes de température de l'ordre de $10^{-6} \mathrm{oK}$.

Si maintenant le tuyau est fermé à la distance $y=L$ par un réflecteur, il s'établit un système d'ondes stationnaires. Si $Z_{L}$ est l'impédance du réflecteur :

$$
V(y)=z_{\infty} q(0) \frac{\mathrm{e}^{-i K(y-L)}-T_{L} \mathrm{e}^{i K(y-L)}}{\mathrm{e}^{i K L}+T_{L} \mathrm{e}^{-i, L L}}
$$

où $T_{L}=\left(z_{\infty}-Z_{L}\right) /\left(z_{\infty}+Z_{L}\right)$ est ce qu'on appelle le facteur de réflexion. Avec un réflecteur parfait $\left(Z_{L}=\infty\right)$, c'est-à-dire ici avec un très bon isolant thermique, on a $T_{L}=-1$. Cela sera pratiquement vérifié dans nos expériences même si le bouchon isolant est recouvert d'un bolomètre de faible capacité thermique (cf. $\S \mathrm{IV}$ ) destiné à mesurer $\Delta T=V(L)$ :

$$
V(L)=z_{\infty} q(0) \frac{2}{\mathrm{e}^{i K L}-\mathrm{e}^{-i K L}} .
$$

On se placera à la résonance lorsque :

$$
L=n \frac{\lambda}{2}=\frac{n \pi u}{\omega_{n}} ; \quad K L=n \pi-i \alpha L
$$

$\alpha$ supposé faible mais différent de zéro limite l'amplitude de la résonance :

$$
V(L)=z_{\infty} q(0) \frac{(-1)^{n}}{\alpha L}
$$

$1 / \alpha L$ est l'amplification à la résonance. Si d'autre part le facteur de qualité $Q$ est défini d'après les mesures pratiques comme le rapport :

$$
Q=\omega_{n} / \Delta \omega
$$

où $\Delta \omega$ est l'écart à la résonance tel que l'amplitude est divisée par $\sqrt{2}$, on a la relation :

$$
1 / \alpha L=Q / n \pi \text {. }
$$

L'impédance ramenée à l'entrée à la résonance est $z=z_{\infty} / \alpha L=z_{\infty} Q / n \pi$.

IV. Dispositif expérimental. - L'échantillon est une lame d'un alliage de plomb et d'indium (In 2,6\%), qui est un supraconducteur de deuxième espèce, devenant normal à $B c_{2} \simeq 2100 \mathrm{G}$ à $1,6 \mathrm{oK}$. La lame a été découpée par étincelage à partir d'un monocristal et polie chimiquement. Ses dimensions sont $20 \times 6$ $\times 1 \mathrm{~mm}(e=1 \mathrm{~mm}, f i g .1)$. Le champ magnétique (direction $z$ ) est donné par deux bobines de Helmholtz en fil supraconducteur (homogénéité $10^{-3}$ sur un volume de l'ordre de $\left.1 \mathrm{~cm}^{3}\right)$.

Il n'existe pas dans la littérature de données sur la résistance Kapitza des alliages de plomb. Il reste que l'ordre de grandeur de $R_{\mathrm{K}}$ est à peu près le même pour tous les métaux entre 1 et $2{ }^{\circ} \mathrm{K}$. Il est à noter que $R_{\mathrm{K}}$ peut varier sensiblement dans la transition normal-supraconducteur. Ainsi, pour le plomb, Challis [12], d'après des mesures en continu, indique une variation de $R_{\mathrm{K}}$ de 2 à $5 \mathrm{oK} \mathrm{cm}^{2} / \mathrm{W}$; cependant, le même auteur ne retrouve plus cette différence en utilisant une technique de deuxième son [13]. Pour éviter une variation éventuelle et mal connue de $R_{\mathrm{K}}$, nous avons doré électrolytiquement la surface de l'échantillon, ce qui doit maintenir la valeur constante de l'or à $1,6 \mathrm{oK}\left(R_{\mathrm{K}} \simeq 2 \mathrm{oK} \mathrm{cm} \mathrm{cm}^{2} / \mathrm{W}[14]\right)$. Le résonateur est un tuyau en araldite de section rectangulaire $6 \times 12 \mathrm{~mm}$, fermé d'un côté par la lame et 
de l'autre par un bouchon coulissant en épibond ( $\left.{ }^{\mathbf{4}}\right)$. Toutes les mesures rapportées ici ont été faites à $1,64 \mathrm{oK}$; la longueur du tuyau est de 3 à $4 \mathrm{~cm}$ et il résonne sur sa fréquence fondamentale, environ $300 \mathrm{~Hz}$.

Comme la vitesse du deuxième son varie avec la température, il est nécessaire, pour assurer la parfaite stabilité de la résonance, de réguler rigoureusement la température du bain. La régulation, à $10^{-4} \mathrm{o} K$ près, est assurée par un chauffage compensateur : une résistance au carbone plongée dans l'hélium, qui est sensible à la température $(R=32 \mathrm{k} \Omega ;-(1 / R)(\mathrm{d} R / \mathrm{d} T)$ $\left.=0,35{ }^{\circ} \mathrm{K}^{-1}\right)$, est mesurée par un pont $\mathrm{ML} 155 \mathrm{AC}$; le déséquilibre du pont pilote un appareil Cryomatik ML 1300, qui alimente en courant continu une résistance chauffante.

De plus, la température de travail $1,64{ }^{\circ} \mathrm{K}$ a été choisie parce qu'elle correspond à un maximum très plat de $u(T)$. La température est mesurée à partir de la pression de vapeur (manomètre à huile).

Le bolomètre détecteur, destiné à mesurer $\Delta T$ dans le plan $y=L[8]$, est une fine couche de graphite $\left(^{5}\right)$ peinte sur le bouchon en épibond; il est essentiel que la matière du support ait sensiblement la même dilatation thermique que la couche de graphite. La résistance du détecteur utilisé est $24,5 \mathrm{k} \Omega$ à $1,6 \mathrm{oK}$ et son coefficient de température $(1 / R)(\mathrm{d} R / \mathrm{d} T)$ est égal à $-0,18 \mathrm{oK}^{-1}$.

L'impédance de fermeture $Z_{L}$ (fig. 3) peut se représenter par une résistance de contact $R_{\mathrm{c}}$ du type

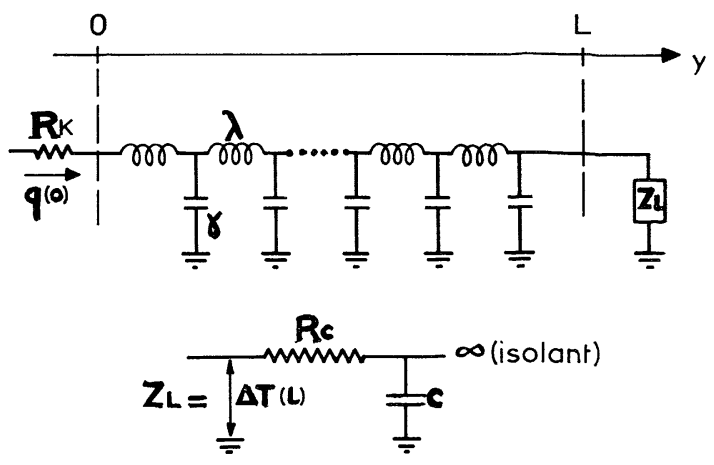

FIG. 3. - Schéma électrique analogue d'un tuyau de longueur $L$.

Kapitza, en série avec une capacité très faible c. L'épaisseur de la couche de carbone est faible devant la profondeur de peau thermique $\delta$ et on peut l'assimiler globalement à une capacité. $Z_{L}=R_{\mathrm{c}}+1 / i \omega c$ est très grande devant $z_{\infty}$ (cf. $\S$ III) et on peut admettre pratiquement que la réflexion est parfaite $\left(T_{L}=-1\right)$. Le bolomètre mesure strictement la « tension aux bornes »de cette capacité, mais c'est pratiquement

$\left(^{4}\right)$ Résine thermodurcissable Epibond 100 A (Furane Plastics, Los Angeles).

${ }^{(5)} 70^{\circ} \prime$ aquadag, suspension colloïdale de graphite, et $30 \%$ Paralac (Imperial Chemical Industries).
$\Delta T(L)$ tant que $1 / \omega c \gg R_{\mathrm{c}}$. Avec des couches de graphite de quelques microns, on peut monter jusqu'à $40 \mathrm{kHz}$.

Un courant continu $I_{0}=60 \mu \mathrm{A}$ fourni par une batterie de piles « polarise » le bolomètre; une résistance de quelques $M \Omega$ placée en série assure un courant constant. L'onde thermique $\Delta T(L) \mathrm{e}^{i \omega t}$ module la résistance $R$ du bolomètre et il apparaît une tension alternative à ses bornes $s \mathrm{e}^{i \omega t}$ :

$$
s=I_{0} \frac{\mathrm{d} R}{\mathrm{~d} T} \Delta T(L)
$$

Le signal $s$ est envoyé dans deux détections synchrones PAR H-R 8, qui mesurent les deux composantes $s_{x}$ et $s_{y}$ de son amplitude complexe, respectivement en phase et en quadrature de phase avec un même signal de référence, qui est par exemple luimême à la phase du courant $j_{x}$ injecté dans l'échantillon. Les deux signaux de sortie sont envoyés, avec la même sensibilité, sur les entrées $\mathrm{X}$ et $\mathrm{Y}$ d'un enregistreur XY Moseley $7000 \mathrm{AM}$; on obtient ainsi un enregistrement direct du signal $s$, en module et en phase, par exemple en fonction du champ magnétique appliqué $B$; ainsi, la figure 4 montre un enregistrement de $s(B)$ pour un échantillon doré de PbIn

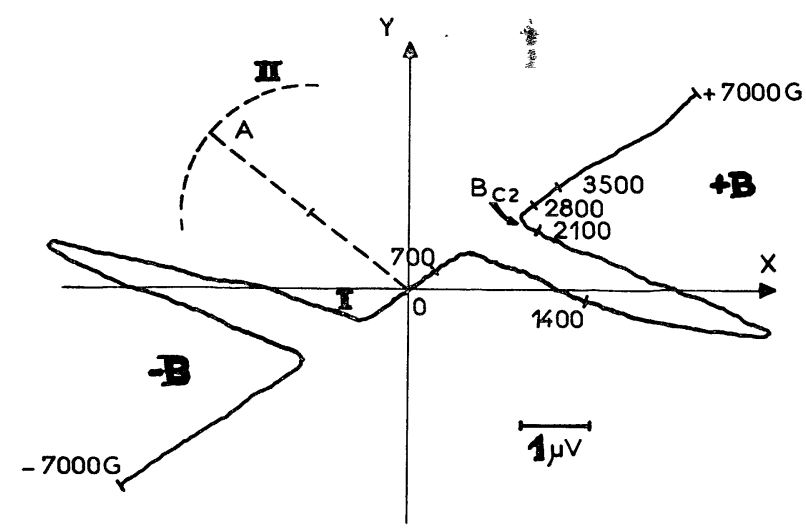

FIG. 4. - Enregistrement dans le plan complexe de l'effet Ettingshausen alternatif $(\omega=335 \mathrm{C} / \mathrm{s})$ dans un échantillon $\mathrm{PbIn}\left(2,6 \%\right.$ In) à $1,6{ }^{\circ} \mathrm{K}$ (courbe $\mathrm{I}$ ). OA correspond à un effet Joule croisé $(5 \mathrm{~A}(\omega)+1 / 2 \mathrm{~A}$ (continu)). La courbe II représente la courbe de résonance à $335 \mathrm{C} / \mathrm{s}$.

à $T=1,64 \mathrm{oK}$, parcouru par un courant alternatif $I_{x} \mathrm{e}^{i \omega t}$ à $\omega=335 \mathrm{~Hz}$, d'amplitude constante $I_{x}=5 \mathrm{~A}$ $\left(j_{x} \simeq 10^{2} \mathrm{~A} / \mathrm{cm}^{2}\right)$.

Un enregistrement se déroule de la manière suivante. A champ nul, $B=0$, on applique un courant $I_{x}(\omega)$, à une fréquence voisine de la fréquence de résonance calculée. Il apparaît toujours un signal parasite, de l'ordre de quelques $\mu \mathrm{V}$, ne serait-ce que par le flux induit dans la boucle de mesure par le fort courant $I_{x}$. Si $I_{x}$ est constant, ce signal parasite ne varie pas; on peut le compenser par les remises à zéro des 
appareils de mesure, et prendre son extrémité comme nouvelle origine ( fig. 4).

Ensuite, le champ $B$ est monté jusqu'à ce que le métal soit dans l'état normal (état dont on connaît la résistivité). Un courant continu $I_{0}$ est superposé au courant $I_{x}(\omega)$ de façon à créer un effet Joule croisé à $\omega$ (source $g_{1}$, cf. $\S$ III). Un signal de deuxième son très net apparaît aux bornes du récepteur et on règle la fréquence $\omega$ de façon à se trouver au voisinage du maximum de la résonance. L'effet Joule croisé permet d'accorder aisément le tuyau à la fréquence du courant et de vérifier la qualité de la résonance; il représente en plus un étalon bien contrôlé de flux de chaleur.

On trouve un facteur $Q$ de l'ordre de 125 , d'où un facteur d'amplification voisin de 40 . Lorsque $\omega$ varie autour de la résonance, on peut admettre que le flux de chaleur $q(0)$ à l'entrée du tuyau est constant, puisque l'impédance équivalente $z$ à l'entrée est au maximum $40 z_{\infty}$, soit environ $4 / 100$ de la résistance Kapitza.

Le signal $\overline{\mathrm{OA}}$ ( fig. 4) est obtenu en superposant à un courant alternatif existant de $0,5 \mathrm{~A}$ un courant continu de $5 \mathrm{~A}$, ce qui crée un effet Joule :

$$
g_{1} e \simeq 0,68 \times 10^{-3} \mathrm{~W} / \mathrm{cm}^{2} .
$$

$\overline{\mathrm{OA}}=3,6 \mu \mathrm{V}$ correspond à un $\Delta T(L)$ sur le bolomètre de l'ordre de $1,4 \times 10^{-5} \mathrm{oK}$, donc à un flux $q(0)$ incident (formule (8)) de $0,17 \mathrm{~mW} / \mathrm{cm}^{2}$, qui ne serait égal à $g_{1} e / 2$ que s'il n'y avait pas de filtrage par effet de peau thermique (ici $\delta \sim e$ ). On vérifie que les conditions de résonance sont stables en s'assurant que la position du point A n'a pas varié avant et après l'enregistrement.

$I_{0}$ étant nul, on mesure uniquement l'effet Ettingshausen puisque tout effet Joule est à $2 \omega$, avec éventuellement des harmoniques pairs supérieurs quand la caractéristique courant-tension de l'échantillon n'est pas linéaire (ce qui est le cas entre $B c_{1}$ et $B c_{2}$ ). Le pic qui se détache sur la courbe $s(B)$ est lié au transport d'entropie dans le mouvement des tourbillons et s'écroule à $B=B c_{2}$; son ordre de grandeur est bien conforme aux résultats des mesures en continu [1]. Par contre, ce pic semble se superposer à un autre signal démarrant à champ nul; or, l'effet Ettingshausen est théoriquement absent avant $B c_{1}$, et pour le métal normal il est généralement beaucoup plus faible. Il s'agit bien cependant d'un effet thermique, fonction impaire du champ continu. Si la température du bain est légèrement déréglée, toutes choses égales ailleurs, le tuyau se trouve désaccordé, et tout le signal se ramasse autour de l'origine. Ce test permet de distinguer nettement un effet thermique de deuxième son de tout autre signal parasite.

On peut envisager dans ce type d'expérience l'éventualité de vibrations mécaniques, dont la phase changerait effectivement de signe avec $B$ comme la force de Laplace. Ces vibrations pourraient alors engendrer, par compressions adiabatiques, un effet thermique secondaire qui apparaîtrait comme un signal parasite dans $s(B)$. Cette hypothèse est cependant tout à fait exclue car il s'agit d'un effet trop faible; il suffit, pour s'en convaincre, de se reporter aux essais infructueux de Shubnikov et Sokolov vers 1941 qui, à la suite des travaux de Landau, tentèrent d'engendrer un deuxième son en plongeant un quartz piézoélectrique dans l'hélium. Lifshitz montra peu après [15] que, dans ces conditions, l'intensité du deuxième son est environ $10^{-6}$ fois plus faible que l'intensité du premier son.

V. Conclusion. - L'avantage de toute technique de modulation avec détection synchrone, sur une méthode en continu, est de permettre la détection de faibles variations d'un signal en fonction d'un paramètre extérieur, par une amélioration du rapport signal sur bruit. D'autre part, les mesures de faibles écarts de température en continu sont particulièrement sensibles à toute dérive de température et aux gradients thermiques accidentels; elles exigent un isolement soigneux soit de tout l'échantillon (calorimétrie), soit de certaines faces seulement (effet thermomagnétique). De plus, ce sont des mesures point par point.

En alternatif au contraire, on permettra un contact avec le bain, et on fera un enregistrement continu du signal. Dans les montages qui sont adoptés généralement $[3,4]$, le flux de chaleur $q_{0}+q_{1} \mathrm{e}^{i \omega t}$ est modulé à une fréquence $\omega$ assez basse (par exemple $10 \mathrm{~Hz}$ ) pour que l'ensemble de l'échantillon, plus le bolomètre collé, plus éventuellement la résistance chauffante (mesures de chaleur spécifique [3]) puissent être considérés comme une capacité unique $C$ où l'on néglige tout effet de peau $\left(C \sim 10^{-6}\right.$ à $\left.10^{-5} \mathrm{~J} / \mathrm{oK}\right)$. Une résistance de fuite très grande $\left(R \sim 10^{5}-10^{6} \mathrm{oK} \mathrm{cm}{ }^{2} / \mathrm{W}\right)$ évacue vers le bain la composante continue $q_{0}$. Si la constante de temps $R C$ (de l'ordre de la seconde) est supérieure à la période de modulation, l'amplitude de la température de $C$ est de l'ordre de $q_{1} \tau / C$ (flux $q_{1}$ pendant une période $\tau$ ). Ici les flux de chaleur imposés sont très faibles (par exemple $10^{-9} \mathrm{~W}$ avec des $\Delta T$ de l'ordre de $10^{-4}-10^{-5} \mathrm{oK}$ ).

Dans une technique de deuxième son, l'échantillon est mis en contact direct avec le bain et pratiquement une grande partie du flux de chaleur est dérivée vers l'hélium. L'impédance de l'hélium étant donnée $\left(z_{\infty} \simeq 2 \times 10^{-3} \mathrm{o} \mathrm{K} \mathrm{cm}^{2} / \mathrm{W}\right)$, et si on opère avec un résonateur ordinaire, dont le facteur d'amplification est de l'ordre de 50 , et un bolomètre courant sensible à $10^{-6} \mathrm{oK}$, les limites de la méthode se traduisent en flux minimum incident $q(0)$; dans l'exemple donné, il faut que $q(0) \gtrsim 10^{-5} \mathrm{~W}$ (cf. [8]). Ainsi, dans les mesures d'absorption par résonance cyclotron de Picard et Carter [4], où la puissance est limitée naturellement à $10^{-9} \mathrm{~W}$, notre méthode est impossible à appliquer. Par contre, en calorimétrie, le chauffage est formé par une résistance auxiliaire, et peut être augmenté dès que le contact avec le bain est meilleur; on pourrait alors envisager de travailler à des fré- 
quences plus élevées que $10 \mathrm{~Hz}$, quitte à tenir compte systématiquement des effets de peau dans une géométrie simple.

Dans le cas des effets thermomagnétiques comme l'effet Ettingshausen, on a vu qu'on pouvait atteindre des flux de chaleur assez importants. Si les différences de température obtenues sont généralement faibles, c'est parce que ce flux débite à travers la très faible résistance thermique de l'échantillon $(\Delta T=R q)$; ainsi pour la lame de $\mathrm{PbIn}$ utilisée, et dans l'état normal, $R=e r=5 \mathrm{~K} \mathrm{~cm}^{2} / \mathrm{W}$. Comme la résistance de Kapitza est du même ordre de grandeur que $R$, une bonne partie du flux est détournée vers l'hélium $(\alpha=1 / 3$ avec $Z=R$ en continu; cf. $\S$ II [6]).

On peut voir dans une technique de deuxième son les avantages suivants : tout d'abord, on peut travailler à des fréquences acoustiques plus élevées qui sont beaucoup plus maniables avec un équipement courant. Dans certains cas, on peut avoir à faire varier la fréquence, qui devient un facteur physique, par exemple pour les supraconducteurs de $2^{\mathrm{e}}$ espèce dans l'état tourbillonnaire. D'autre part, le montage de l'échantillon est plus facile; on peut, avec un même bolomètre, permuter plusieurs échantillons, ou, s'il y a lieu, faire différents traitements de surface. On évite ainsi le réglage d'une résistance de fuite pas trop faible, qui est souvent aussi délicat que l'isolement thermique. Enfin, on a vu comment on pouvait distinguer tout signal thermique issu de l'échantillon et amplifié par le deuxième son de tout autre signal parasite superposé (vibrations mécaniques, tensions induites) surtout si, comme dans l'effet Ettingshausen, l'échantillon doit être traversé par un courant alternatif de plusieurs ampères.

\section{BIBLIOGRAPHIE}

[1] Solomon (P. R.) et OtTer (F. A.), Phys. Rev., 1967, 164, 608 .

[2] McCombe (B. D.) et Seidel (G.), Phys. Rev., 1967 155, 633.

[3] Sullivan (P. F.) et Seidel, (G.), Phys. Rev., 1968, 173, 679 .

[4] Picard (J. C.) et Carter (D.), Proc. of the Int. Conf. on the Physics of Semiconductors, Kyoto, 1966; J. of the Phys. Soc., Japan, 1966, 21, Suppl., 202.

[5] Landau (L.), J. Phys. U.R.S.S., 1941, 10, 389 ; $1944,8,38$.

[6] Peshuov (V.), J. Phys. U.R.S.S., 1944, 8, 381 ; 1946, 10, 389.
[7] Peshrov (V.), JETP (U.R.S.S.), 1948, 18, 867.

[8] KURTr (N.) et MaC INTOSH (J.), Conférence Internationale de Physique des Très Basses Températures, Paris, 1955.

[9] CALLEN (H. B.), Thermodynamics, John Wiley and Sons, N.Y., 1960.

[10] Voir par exemple ATKIns (K. R.), Liquid Helium, Cambridge University Press, 1959.

[11] Dingle (R. B.), Proc. Phys. Soc., 1948, 61, 9.

[12] Challis (L. J.), Proc. Phys. Soc., 1962, 80, 759.

[13] Communication à L.T. 11 (1968), à paraître.

[14] Johnson (R. C.) et LitThe (W. A.), Phys. Rev., 1963, 130, 596

[15] LIFSHITZ (E.), J. Phys. U.R.S.S., 1944, 8, 110. 